\title{
航空发动机压气机叶片外物损伤规律研究 ${ }^{*}$
}

\author{
舒 畅 ${ }^{1}$ 程 铭 ${ }^{1,2}$ 许 煜 ${ }^{1}$ 程 礼 $^{1,3}$ \\ (1. 空军工程大学航空工程学院 西安 710038; \\ 2. 中国人民解放军 93066 部队 牡丹江 157000 ; \\ 3. 先进航空发动机协同创新中心 北京 100191)
}

\begin{abstract}
摘要: 通过对某型航空发动机压气机转子叶片外物损伤数据进行深入分析, 揭示出损伤类型、缺口尺寸和位置沿压气机轴向 和径向的分布规律。研究结果表明: 在缺口、撕裂、凹坑和卷曲四类典型损伤中, 缺口损伤所占的比重最大; 低压压气机损 伤率较高, 且各级叶片损伤率随压气机级数的增加呈现下降趋势, 高压压气机各级叶片中, 各损伤类型表现出显著差异性, 该特点与叶片材料、冲击能量等因素的变化有关; 缺口损伤尺寸沿压气机的轴向分布差异性不明显, 在高、低压气机中满足 不同的分布形式, 且损伤位置仅与深度尺寸存在显著关联; 低压一级叶片大多数缺口损伤位于距离叶根高度 $80 \%$ 以上区域, 其损伤位置与损伤尺寸没有显著关联，但损伤宽度和损伤深度存在线性相关性。
\end{abstract}

关键词: 航空发动机; 压气机叶片; 外物损伤; 损伤特征; 统计分析

中图分类号: V232

\section{Study on Foreign Object Damage Regular Pattern of Aero Engine Compressor Blades}

\author{
SHU Chang ${ }^{1}$ CHENG Ming ${ }^{1,2} \quad \mathrm{XU} \mathrm{Yu}^{1} \quad$ CHENG Li ${ }^{1,3}$
}

(1. Aeronautics Engineering College, Air Force Engineering University, Xi'an 710038;

2. 93066 Unit of People's Liberation Army of China, Mudanjiang 157000;

3. Co-Innovation Center for Advanced Aero-Engine, Beijing 100191)

\begin{abstract}
Based on the analysis of foreign object damage on certain aero engine compressor blades, the distribution regular pattern of the damage type, notch size and position along the axial and radial directions of the compressor is revealed.The results indicate that the notch is the main type of four typical damages which include notches,tears,pits and curls. The damage rates on low pressure compressor blades are relatively high, and they have a downward trend with the increase of compressor stages, while the damage types on high pressure compressor blades are quite different, because of many changing factors such as blade material and impact energy. Furthermore, there is no obvious difference in the distribution of notch sizes along the axial direction of compressors, but their distribution characteristics are quite different between high and low pressure compressors, the damage location only has a significant correlation with the depth sizes. As far as the first blade of the low pressure compressors is concerned, most of notch damages are located more than $80 \%$ of the height above the blade root, there is no significant correlation between the notch position and the size, but there is a linear correlation between the notch width and depth.
\end{abstract}

Key words: aero engine; compressor blade; foreign object damage; damage characteristics; statistical analysis

\section{0 前言}

飞机在起飞、降落以及低空飞行过程中，其发 动机风扇叶片易遭受由高速气流携带的外来物撞击 从而造成损伤，按照外来物的不同，可分为软物撞

* 国家重点基础研究发展计划资助项目(973 计划, 2015CB057400)。 20180716 收到初稿, 20181125 收到修改稿
击和硬物撞击 ${ }^{[1]}$ 。国内外航空发动机设计规范中, 仅将硬物冲击损伤称为 “外物损伤” ${ }^{[2]}$ (Foreign object damage, FOD)。

外物损伤严重威胁航空发动机的运行安全 ${ }^{[3-4]}$ 。 据统计，因外物损伤造成提前翻修的发动机占提前 翻修发动机总数的 $40 \%$ 以上 ${ }^{[5]}$ 。其中, 环境因素和 叶片因素是损伤频繁发生的两大主要原因 ${ }^{[6]}$ 。外来 物在压气机叶片间的冲击与反弹常造成叶片局部机 
械损伤，损伤部位一般会产生应力集中、残余应力 或微小裂纹 ${ }^{[7-8]}$, 微裂纹也会因叶片的高速旋转、高 频振动而快速扩展, 显著降低叶片疲劳强度, 短时 间内即可引起叶片断裂失效 ${ }^{[9-11]}$ 。

对于外物损伤规律的研究, 国外已经取得了一 些成果。文献[12]将 FOD 的损伤形式总结为 “缺口” (含撕裂)和 “凹痕或鼓包” 两类, 并指出损伤分布 于叶盆面的进气边和排气边; 文献[13]总结归纳了 17 种 FOD 损伤形式, 并指出其中 8 种与叶片高频 振动疲劳有关; 美国空气动力实验室 ${ }^{[13]}$ 将损伤形式 大致分为缺口、撕裂、凹痕 3 种, 结果表明: FOD 常见于压气机叶片前缘, 多发生在进气边距叶根 $80 \%$ 以上的区域, FOD 损伤深度的分布区间为 $0.05 \sim 13 \mathrm{~mm}$, 平均值约为 $1.5 \mathrm{~mm}$, 其密度和损伤 程度从叶根至叶尖明显增加。

国内针对发动机叶片硬物损伤规律的统计分析 工作起步较晚, 数据资料较为缺乏, 相关研究仍在 继续。康继东等 ${ }^{[6]}$ 曾对 1135 台某型系列发动机压气 机叶片损伤进行过统计, 结果表明 $30 \%$ 叶片属于打 伤性质, 而砂石打伤又占其中的 $50 \%$, 且以进气边 居多; 潘辉 ${ }^{[14]}$ 指出, 中小尺度的缺口损伤多分布在 叶片进、排气边区域的上部，大尺度的缺口损伤则 集中分布在叶片进气边区域的叶尖部分; 马超等 ${ }^{[15-16]}$ 整理了国内主要航空公司近 20 年来的 FOD 数据, 分析了损伤类型与发动机类型、损伤位置的关系以 及损伤尺寸间的关系。

为了进一步探求压气机外物损伤规律，找到损 伤易发部位、损伤类型分布特点以及损伤特征的联 系, 本文对某型浴扇发动机叶片近年来发生的近百 起 FOD 数据进行深入分析, 进一步揭示了压气机叶 片间各类损伤的分布规律及其关联性。

\section{1 某型发动机压气机结构特点}

本文研究对象为某型小涵道比的加力式双转子 涡轮风扇发动机, 是广泛用于第三代战机的动力装 置。其轴流式压气机分为低压压气机和高压压气机, 其中低压四级, 高压九级, 共十三级。低、高压压 气机转子最大转速分别为 $10098 \mathrm{r} / \mathrm{min}$ 和 13300 $\mathrm{r} / \mathrm{min}$ 。压气机总增压比为 23 , 空气流量 $112 \mathrm{~kg} / \mathrm{s}$ 。 表 1 和表 2 分别为低、高压压气机各级转子主要参 数, 其中叶片线速度范围对应着发动机稳定运行的 速度范围。由两表可知, 除低压第四级转子外, 叶 片数量基本随级数的增加而增多; 压气机叶片长度 随级数增加而减少; 转速一定时, 低压压气机叶尖 线速度随级数的增加而减小，高压压气机叶根线速
度随级数的增加而增大。

表 1 低压压气机各级转子参数

\begin{tabular}{cccccc}
\hline 级数 & 叶片数 & $\begin{array}{c}\text { 内部 } \\
\text { 直径 } \\
\end{array}$ & $\begin{array}{c}\text { 外部 } \\
\text { 直径 } \\
/ \mathrm{mm}\end{array}$ & $\begin{array}{c}\text { 叶根线速度 } \\
\text { 范围 } /(\mathrm{mm} / \mathrm{s})\end{array}$ & $\begin{array}{c}\text { 叶尖线速度 } \\
\text { 范围 } /(\mathrm{m} / \mathrm{s})\end{array}$ \\
\hline 1 & 37 & 365 & 893.3 & $57.90 \sim 192.99$ & $141.69 \sim 472.31$ \\
2 & 45 & 551 & 837.6 & $87.40 \sim 291.33$ & $132.86 \sim 442.86$ \\
3 & 57 & 494.4 & 779.8 & $78.42 \sim 261.40$ & $123.69 \sim 412.30$ \\
4 & 43 & 512 & 778.3 & $81.21 \sim 270.71$ & $123.45 \sim 411.51$ \\
\hline
\end{tabular}

表 2 高压压气机各级转子参数

\begin{tabular}{cccccc}
\hline 级数 & 叶片数 & $\begin{array}{c}\text { 内部 } \\
\text { 直径 } \\
/ \mathrm{mm}\end{array}$ & $\begin{array}{c}\text { 外部 } \\
\text { 直径 } \\
/ \mathrm{mm}\end{array}$ & $\begin{array}{c}\text { 叶根线速度 } \\
\text { 范围 } /(\mathrm{m} / \mathrm{s})\end{array}$ & $\begin{array}{c}\text { 叶尖线速度 } \\
\text { 范围 } /(\mathrm{m} / \mathrm{s})\end{array}$ \\
\hline 1 & 47 & 409 & 600 & $199.38 \sim 284.83$ & \\
2 & 62 & 444.2 & 600 & $216.54 \sim 309.34$ & \\
3 & 73 & 474 & 600 & $231.06 \sim 330.09$ & \\
4 & 94 & 500 & 600 & $243.74 \sim 348.20$ & \\
5 & 99 & 520 & 600 & $253.49 \sim 362.13$ & $292.49 \sim 417.84$ \\
6 & 101 & 532 & 600 & $259.34 \sim 370.48$ & \\
7 & 103 & 542 & 600 & $264.21 \sim 377.45$ & \\
8 & 105 & 548 & 600 & $267.14 \sim 381.63$ & \\
9 & 107 & 553 & 600 & $269.58 \sim 385.11$ & \\
\hline
\end{tabular}

低压压气机各级转子叶片材料为 BT3-1 钛合 金; 高压压气机 $1 、 2 、 3$ 级转子叶片材料为 BT3-1 钛合金, $4 、 5$ 级转子叶片材料为 BT $18 \mathrm{y}$ 热强钛合 金, $6 、 7 、 8 、 9$ 级转子叶片材料为 ЭП-718ИД 高 温合金。

\section{2 叶片外物损伤形式及其分布}

损伤形式的不同是损伤程度差异的表现，反映 了叶片材料、外来物、冲击角度、速度等诸多因素 对损伤构成的影响。统计损伤在各级压气机间的分 布状况，进而分析损伤类型与压气机类型间差异性， 有助于判断压气机中主要损伤类型以及预测损伤情 况等，为叶片损伤早期诊断提供依据。

\section{1 损伤形式的分类}

通过对损伤叶片的统计分析, 将具有典型特征 的叶片硬物冲击损伤分为以下四类：缺口、撕裂、 凹坑和卷曲。其中缺口表现为叶片局部材料缺失, 形成具有一定宽度和深度的孔洞; 撕裂表现为叶片 受冲击剪切力作用形成的裂缝, 可用裂缝长度描述 损伤程度; 凹坑表现为叶片受冲击形成局部凹陷, 损伤部位有明显压痕; 卷曲表现为叶片边缘处材 料发生弯折、褶皱等扭曲变形, 各损伤类型如图 1 所示。 


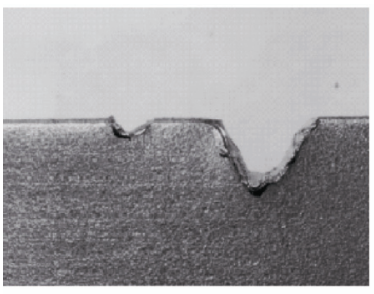

(a) 缺口

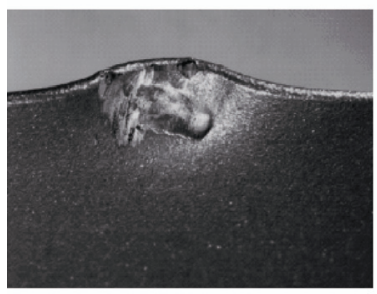

(c) 凹坑

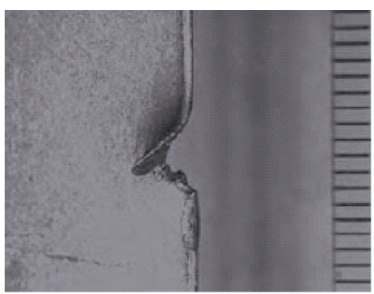

(b) 撕裂

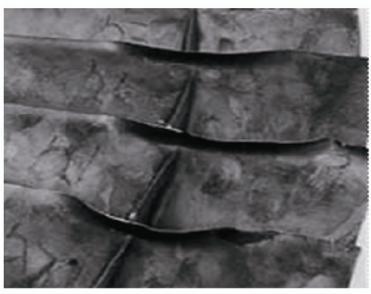

(d) 卷曲
图 1 四类典型损伤

图 2 表示出各损伤类型所占比重的情况, 其中 缺口占总损伤比重为 $83 \%$, 为主要损伤类型, 其次 为卷曲, 凹坑和撕裂所占的比重均较少。

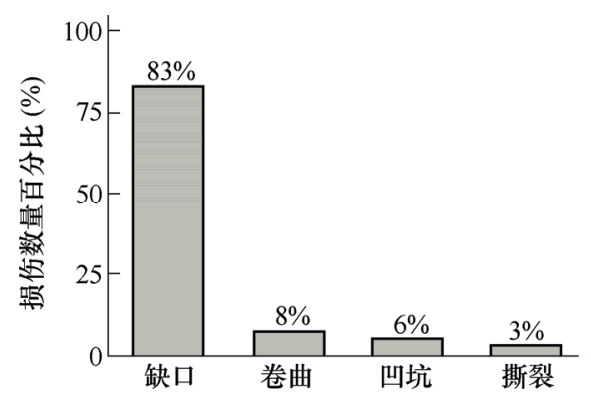

图 2 各类典型损伤所占的百分比

\section{2 损伤形式在高低压气机间的分布状况}

考虑到不同压气机类型中叶片数量的影响, 将损伤数量除以压气机叶片数量定义为相对损伤 率, 得到低压压气机叶片损伤率为 0.148 , 高压压 气机叶片损伤率为 0.077 , 表明低压压气机损伤率 较高。

利用 SPSS 交叉表对损伤类型与压气机类型进 行差异性分析, 两者属于名义变量, 进行卡方检验, 得似然比显著性水平为 $0.065>0.05$, 表明高、低压 压气机的损伤类型不存在显著差异性。表 3 反映了 各损伤类型在高、低压气机中的比例, 可见高、低 压气机中的主要损伤为缺口损伤, 其他类型的损伤 所占的比重均较小。

表 3 各损伤类型在压气机中的比例 $\%$

\begin{tabular}{|c|c|c|c|c|c|c|c|}
\hline \multicolumn{4}{|c|}{ 低压压气机损伤类型 } & \multicolumn{4}{|c|}{ 高压压气机损伤类型 } \\
\hline 缺口 & 撕裂 & 凹坑 & 卷曲 & 缺口 & 撕裂 & 凹坑 & 卷曲 \\
\hline 89 & 7 & 4 & 0 & 80 & 2 & 7 & 11 \\
\hline
\end{tabular}

低压压气机各级叶片损伤率如表 4 所示。将各 级叶片损伤率的比重绘制如图 3 所示, 可见第一级
叶片损伤率比重达 $66 \%$, 前三级叶片损伤率比重高 达 $97 \%$, 且叶片损伤率随级数的增加呈现出递减趋 势, 可以推断外来物与低压叶片碰撞的冲击动能随 级数的增加而减小, 这可能与外来物尺寸(发生破 裂)、冲击速度、角度等因素变化相关。

同理，对损伤类型与低压压气机叶片级数进行 差异性分析, 得显著性水平为 $0.102>0.05$, 表明低 压各级叶片的损伤类型不具有显著差异性。

表 4 低压压气机各级叶片损伤率

\begin{tabular}{cc}
\hline 叶片级数 & 损伤率 \\
\hline 1 & 0.432 \\
2 & 0.133 \\
3 & 0.070 \\
4 & 0.023 \\
\hline
\end{tabular}

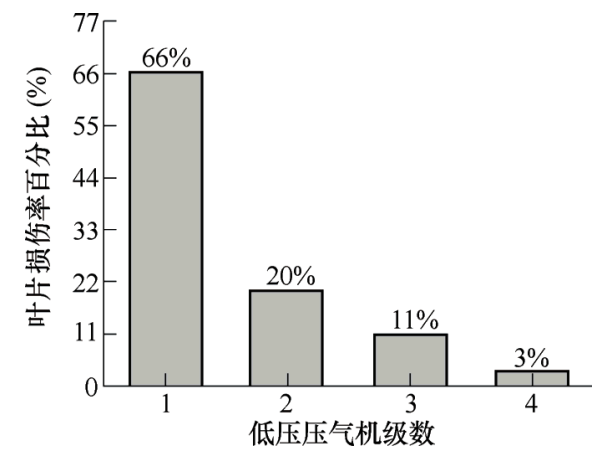

图 3 低压压气机各级叶片损伤率所占百分比

高压压气机各级叶片损伤率如表 5 所示, 其各 级叶片损伤率不存在随级数增加而递减的规律。

表 5 高压压气机各级叶片损伤率

\begin{tabular}{cc}
\hline 叶片级数 & 损伤率 \\
\hline 1 & 0.106 \\
2 & 0.065 \\
3 & 0.096 \\
4 & 0.085 \\
5 & 0.040 \\
6 & 0.069 \\
7 & 0.068 \\
8 & 0.114 \\
9 & 0.065 \\
\hline
\end{tabular}

同理，对损伤类型与高压压气机叶片级数进行 差异性分析, 显著性水平为 $0.031<0.05$, 表明高压 压气机各级叶片中的损伤类型具有显著差异。图 4 反映了这种差异性, 高压压气机前几级叶片通常只 出现缺口损伤且其损伤率普遍较高, 撕裂、凹坑主 要集中在中间级叶片上且损伤率保持在低水平，卷 曲发生在第八级叶片上, 发生该损伤时损伤率较高。 高压压气机损伤类型随级数增加依次出现缺口、撕 裂、凹坑、卷曲等形式，与叶片材料以及外来物冲 
击能量的变化有着密切联系。外来物在刚进入高压 压气机时具有较高的速度，与前三级 BT3-1 钛合金 叶片碰撞时只造成了缺口损伤; 部分外来物发生碰 撞后能量降低, 与抗破坏性更强的第 4、5 级 BT18y 钛合金叶片碰撞时开始出现撕裂、凹坑，且缺口损 伤率明显降低; 最后与末四级高温合金叶片碰撞, 缺口、凹坑损伤率较为稳定。

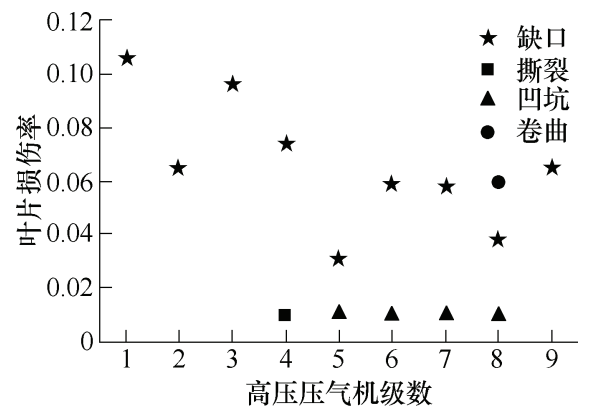

图 4 高压压气机各级叶片中各类损伤的损伤率

\section{3 缺口损伤尺寸分析}

缺口损伤为损伤的主要类型, 因而需引起研究 的足够重视。根据缺口损伤形貌特征, 可用缺口的 宽度和深度来描述损伤程度。

\section{1 低压压气机叶片缺口尺寸分析}

低压压气机叶片缺口宽、深尺寸特征如表 6 所 示，其缺口宽度尺寸均值高于深度尺寸。

\section{表 6 低压压气机叶片缺口宽度与深度}

\begin{tabular}{ccccccc}
\hline \multicolumn{3}{c}{ 缺口宽度 $/ \mathrm{mm}$} & & \multicolumn{3}{c}{ 缺口深度 $/ \mathrm{mm}$} \\
\cline { 1 - 1 } \cline { 5 - 7 } 平均值 & 标准差 & 方差 & & 平均值 & 标准差 & 方差 \\
\hline 3.280 & 1.744 & 3.041 & & 2.892 & 1.925 & 3.705 \\
\hline
\end{tabular}

将尺寸数据分别进行整理, 绘制出图 5、6, 可见宽、深尺寸范围均分布在 $0 \sim 8 \mathrm{~mm}$ 区间内, 累积曲线表明缺口数量在宽度 $1 \sim 6 \mathrm{~mm}$ 区间内有 较大累积量，且该区段每个小区间的增长率接近; 缺口数量在深度小于 $4 \mathrm{~mm}$ 区间内有较高的累积 速率。

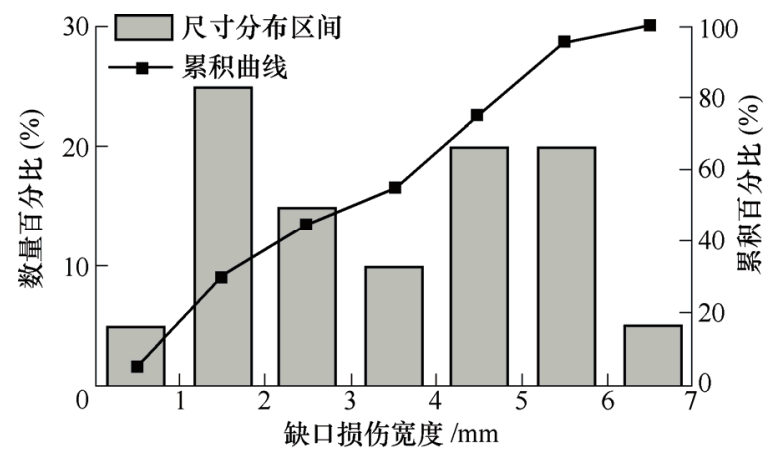

图 5 低压压气机叶片缺口损伤宽度尺寸分布

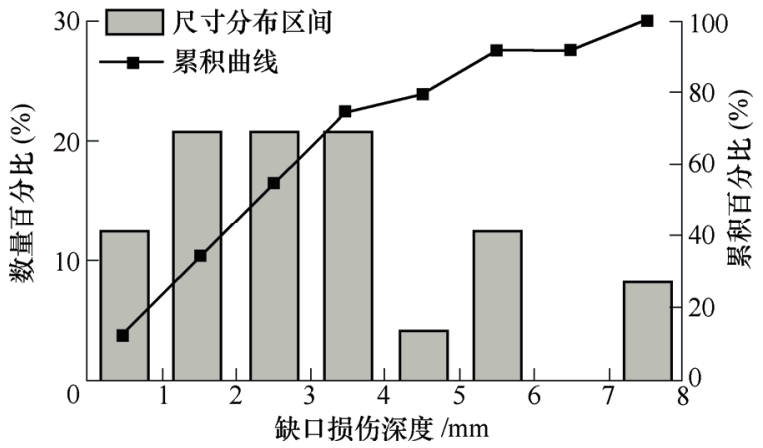

图 6 低压压气机叶片缺口损伤深度尺寸分布

图 7 为缺口宽、深尺寸正态 Q-Q 图(QuantileQuantile Plot), 两者样本值和正态分布参考线拟合 得较好。分别用单样本 $\mathrm{K}-\mathrm{S}$ 非参数检验法 ${ }^{[17]}$ (Kolmogorov-Smirnov)检验两者的分布形态, 得到宽 度 检验概率为 $0.139>0.05$, 深度检验概率为 $0.173>0.05$, 从而验证了两者满足正态分布。

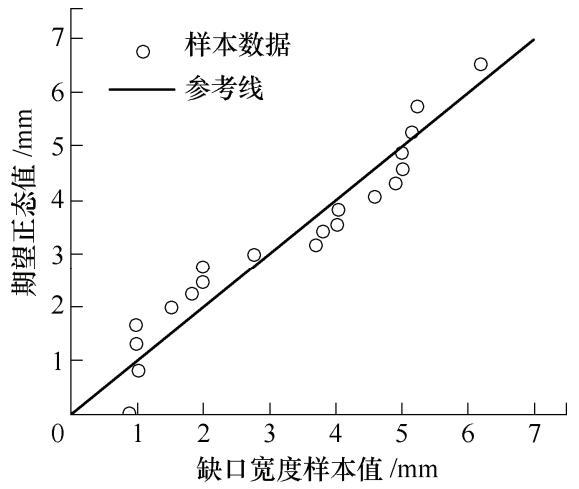

(a)

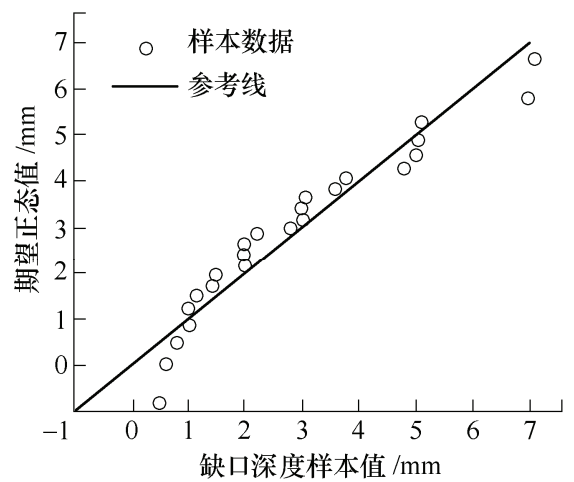

(b)

图 7 低压压气机叶片缺口损伤尺寸正态 Q-Q 图

基于缺口宽、深尺寸的正态分布类型, 对两者 进行 Pearson 相关性分析 ${ }^{[18]}$, 计算得相关系数 $r=0.2$, 显著性水平 $p=0.397>0.05$, 表明低压压气机叶片缺 口宽、深尺寸间不存在显著相关性。散点图 8 直观 地反映了上述结论，从该图中还可以发现散点的分 布存在两个较为集中的区域。进一步对缺口宽度与 深度尺寸进行 K-means 聚类分析 ${ }^{[19]}$, 将它们分为 两个类别。从表 7 可以看出, 第一类中缺口宽度均 值为 $1.40 \mathrm{~mm}$, 对应的深度均值为 $1.87 \mathrm{~mm}$; 第二 
类中缺口宽度均值为 $4.69 \mathrm{~mm}$, 对应的深度均值为 $3.16 \mathrm{~mm}$ 。

\section{表 7 低压压气机叶片缺口宽度与深度聚类分析}

\begin{tabular}{ccc}
\hline 类别 & 缺口宽度平均值 $/ \mathrm{mm}$ & 缺口深度平均值 $/ \mathrm{mm}$ \\
\hline 1 & 1.40 & 1.87 \\
2 & 4.69 & 3.16 \\
\hline
\end{tabular}

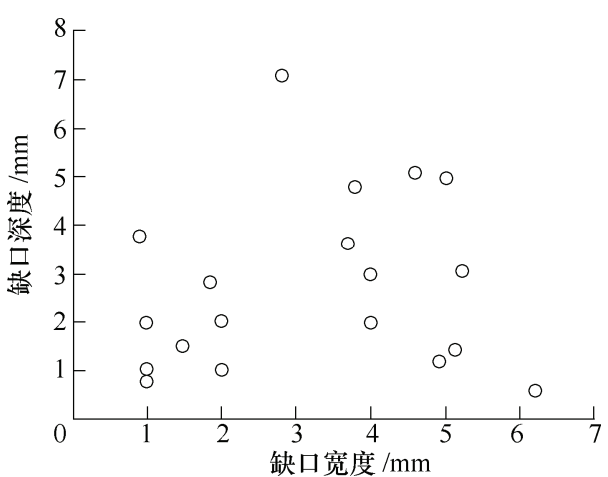

图 8 低压压气机叶片缺口宽度与深度散点图

缺口的宽、深尺寸可视为以叶片级数作为分组 变量的两个样本组, 可采用 Jonckheere-Terpstra $(\mathrm{J}-\mathrm{T})$ 分组检验算法对低压压气机级数及缺口损伤尺寸的 差异性检验, 得到级数与损伤宽度间的检验概率 $p=0.407>0.05$, 而其与损伤深度间的检验概率 $p=0.141>0.05$, 这表明低压压气机各级叶片的缺口 损伤尺寸没有表现出显著差异性。

\section{2 高压压气机叶片缺口尺寸分析}

高压压气机叶片缺口宽、深尺寸特征如表 8 所 示, 其缺口宽度尺寸均值也大于深度尺寸, 但深度 尺寸分散性较小。

\section{表 8 高压压气机叶片缺口宽度与深度}

\begin{tabular}{ccccccc}
\hline \multicolumn{3}{c}{ 缺口宽度 $/ \mathrm{mm}$} & & \multicolumn{3}{c}{ 缺口深度 $/ \mathrm{mm}$} \\
\cline { 1 - 3 } \cline { 5 - 7 } 平均值 & 标准差 & 方差 & & 平均值 & 标准差 & 方差 \\
\hline 2.817 & 1.776 & 3.154 & & 1.558 & 1.113 & 1.240 \\
\hline
\end{tabular}

将尺寸数据分别进行整理, 绘制出图 9、图 10, 可见宽度尺寸的分布范围较深度尺寸广, 累积曲线 表明缺口数量在宽度为 $1 \sim 6 \mathrm{~mm}$ 区间内几乎达到了

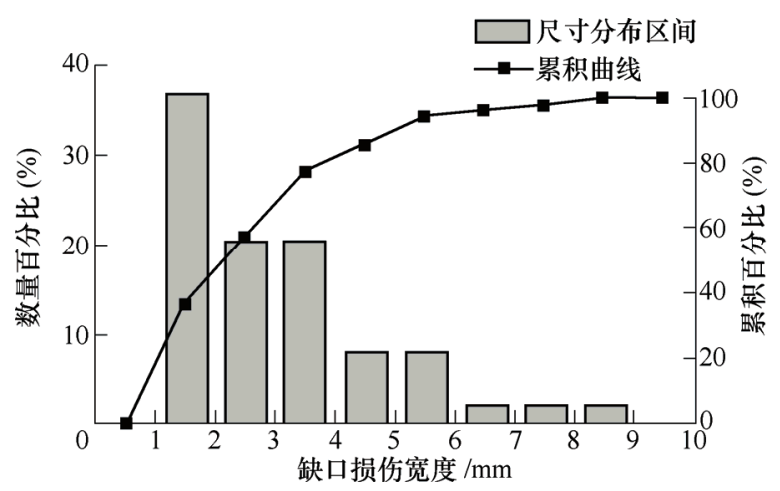

图 9 高压压气机叶片缺口损伤宽度尺寸分布

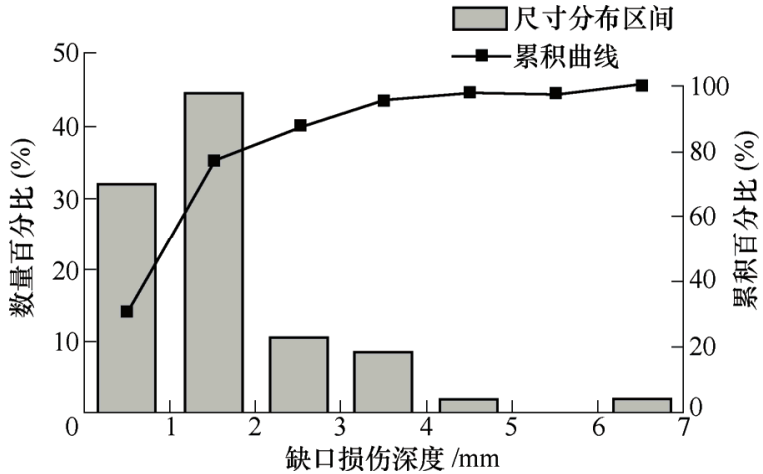

图 10 高压压气机叶片缺口损伤深度尺寸分布

累积总量, 且累积速率在各区间逐渐递减; 缺口数 量在深度小于 $4 \mathrm{~mm}$ 区间内也几乎达到了累积总量,

且累积速率也存在递减趋势。

将缺口宽、深度数据分别绘制于多种类型 $\mathrm{Q}-\mathrm{Q}$ 图进行比较, 结果表明两者样本值和对数正态分布 参考线拟合得较好, 如图 11 所示。分别用单样本 $\mathrm{K}-\mathrm{S}$ 非参数检验法进行检验, 得到宽度检验概率为 $0.09>0.05$, 深度检验概率为 $0.178>0.05$, 从而验证 了两者满足对数正态分布。宽、深尺寸对数正态分 布尺度参数分别为 $2.384,1.274$; 形状参数分别为 $0.573,0.627$ 。

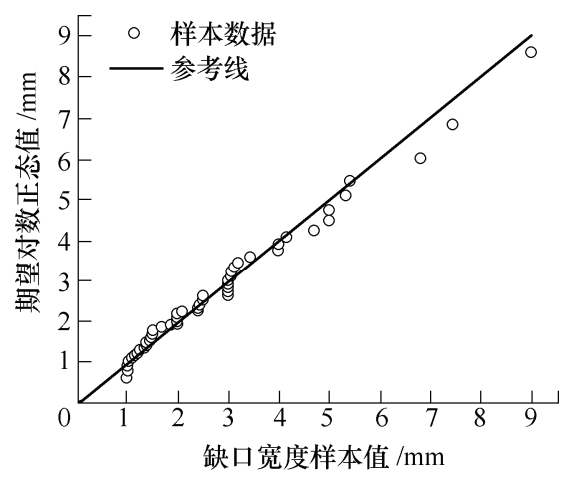

(a)

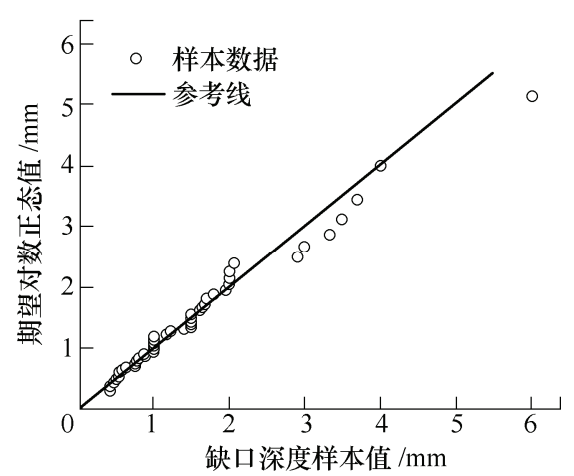

(b)

图 11 高压压气机叶片缺口损伤对数正态 Q-Q 图

基于缺口宽、深尺寸的非正态分布，对两者进 行 Spearman 相关性分析 ${ }^{[18]}$, 得到相关性系数 $r=0.6$, 检验概率 $p$ 接近 $0<0.05$, 故高压压气机叶片缺口宽 度与深度尺寸间存在显著相关性。根据上述分析结 
果, 将尺寸取对数, 绘制成散点图 12。该图直观地 反映出两者具有线性相关性。对两者的线性关系进 行拟合，在散点图中心区域的拟合直线为

$$
y=-0.314+0.64 x
$$

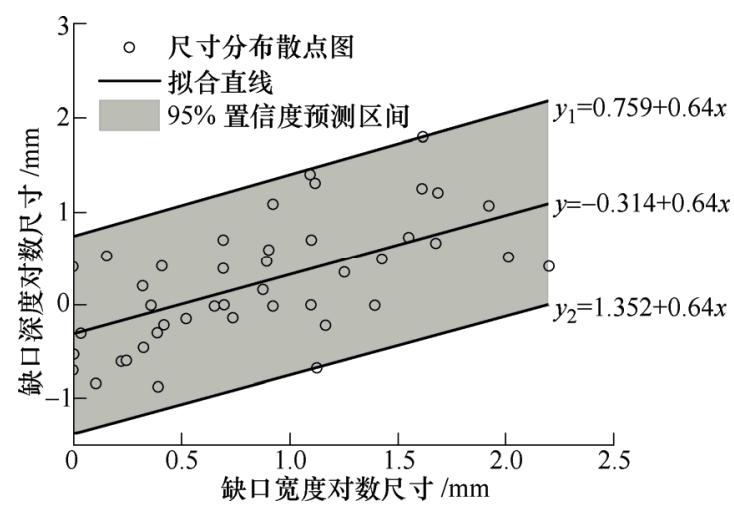

图 12 高压压气机叶片缺口宽度与深度散点图

由于各数据点距该直线的偏差较大, 拟合决定 系数 $R^{2}$ 仅为 0.34 , 拟合残差最值为 -1.038 和 1.073 , 直接判断两者对数尺寸满足式(1)的线性关系误差 较大。但由图可知散点基本位于在 $95 \%$ 置信度的预 测区间内，区间界限为直线

$$
\begin{gathered}
y_{1}=0.759+0.64 x \\
y_{2}=-1.352+0.64 x
\end{gathered}
$$

可以推测缺口深度及宽度的对数尺寸构成的散 点大致分布在 $y_{1}$ 和 $y_{2}$ 这两条直线之间, 并在直线 $y$ 附近波动。

同样用 $\mathrm{J}-\mathrm{T}$ 算法对高压压气机级数及缺口损伤 尺寸的差异性检验, 得到级数与损伤宽度、损伤深 度间的检验概率分别为 $0.702,0.802$, 均大于 0.05 , 表明高压压气机各级叶片的缺口损伤尺寸也没有表 现出显著差异性。

\section{3 缺口损伤位置与损伤尺寸分析}

用缺口损伤距叶根高度的百分比来表述损伤的 相对位置, 并用单样本 K-S 检验算法检验其分布形 态, 结果表明缺口相对位置不满足正态分布, 故需 要采用 Spearman 相关性检验算法对其与缺口损伤 尺寸进行相关性分析。

相关性检验得到, 低压压气机缺口损伤相对位 置及损伤宽度检验概率 $p=0.319>0.05$, 而其与损伤 深度检验概率 $p=0.021<0.05$ 。高压压气机缺口损伤 相对位置及损伤宽度相关性的检验概率 $p=0.525>0.05$, 而其与损伤深度检验概率 $p=0.022<0.05$ 。

检验结果表明, 高、低压气机缺口相对位置和 缺口宽度没有显著相关性, 但其与缺口深度存在显 著相关性。从发动机运行的实际情况考虑, 缺口损 伤宽度主要由外来物尺寸大小决定, 外来物尺寸和
撞击位置之间没有明显的联系，从而造成缺口宽度 和缺口位置之间没有相关性; 而缺口损伤深度不仅 由外来物尺寸决定，而且和叶片旋转的速度大小密 切相关。叶片工作时，沿叶高不同位置具有不同的 转速，靠近叶尖位置线速度大，一般而言产生的损 伤深度越深。

将缺口相对位置和缺口深度的散点图绘制如图 13 和图 14, 两图共同反映出缺口损伤越靠近叶尖, 损伤尺寸的分散性越大; 相对位置所对应损伤深度 最大值基本随着位置的升高而增大，位置与深度间 具有一定的正相关性。图 13 所示, 低压压气机叶片 在相对位置为 0.9 以上的区域集中了大多数缺口, 且它们的深度尺寸分布范围广，分散性大；而在高 压压气机中，如图 14 所示，缺口在相对位置为 0.4 以上的区域都有所分布, 且深度尺寸主要集中在 $0.5 \mathrm{~mm}$ 到 $2 \mathrm{~mm}$ 之间。

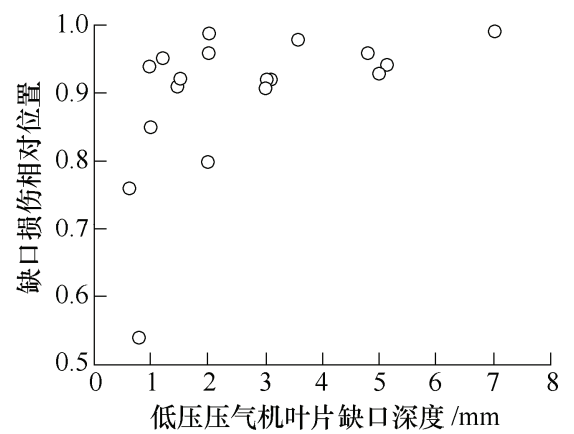

图 13 低压压气机叶片缺口相对位置与深度散点图

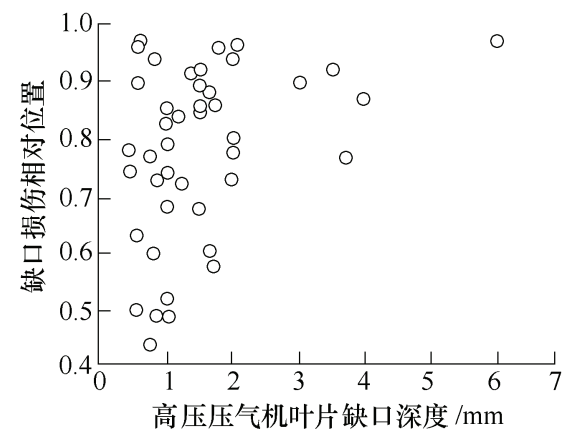

图 14 高压压气机叶片缺口相对位置与深度散点图

\section{4 低压一级叶片缺口损伤分析}

低压一级叶片位于压气机叶片的最前端, 最容 易与外来物发生撞击且对外物损伤最为敏感，因此 对该叶片的损伤分析具有典型性和代表性。

\section{1 低压一级叶片缺口损伤位置分布}

低压一级叶片缺口损伤位置分布如图 15 所示, 大多数损伤距离叶根高度 $80 \%$ 以上，损伤个数在距 叶根 $90 \%$ 以上区间内的累积速率较高。 


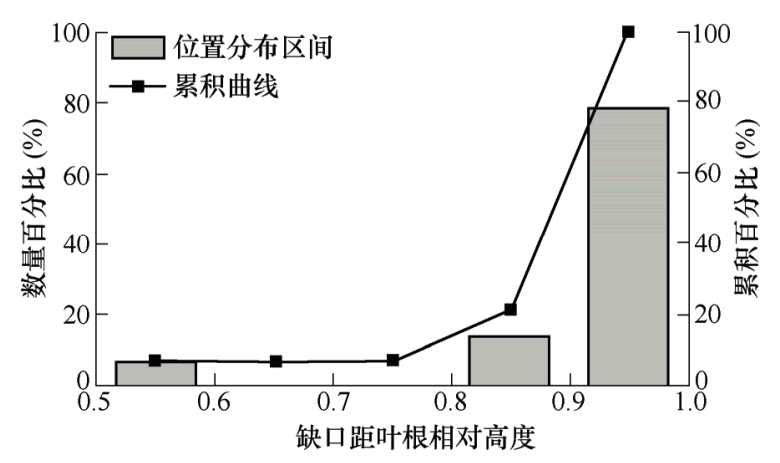

图 15 低压一级叶片缺口损伤沿叶高数量分布

\section{2 低压一级叶片缺口损伤分析}

用单样本 K-S 检验算法得到低压一级叶片缺口 位置不满足正态分布, 而其宽、深尺寸满足正态分 布。故采用 Spearman 算法检验位置与损伤尺寸相关 性，而采用 Pearson 算法检验宽、深尺寸相关性。

Spearman 相关性检验得到, 缺口损伤相对位置 及损伤宽度相关性的检验概率 $p=0.661>0.05$, 两者 没有显著相关性; 损伤相对位置与损伤深度相关性 的检验概率为 $p=0.116>0.05$, 两者也不存在显著相 关性。

Pearson 相关性检验得到, 缺口损伤宽、深尺寸 相关性的检验概率 $p=0.001<0.05$, 存在显著相关性, 且相关系数 $r=0.846$, 正相关程度较高。从两者散点 图 16 可见, 散点的分布同样存在两个较为集中的区 域。因此对两者进行聚类分析, 可以分为两类, 情 况如表 9 所示。

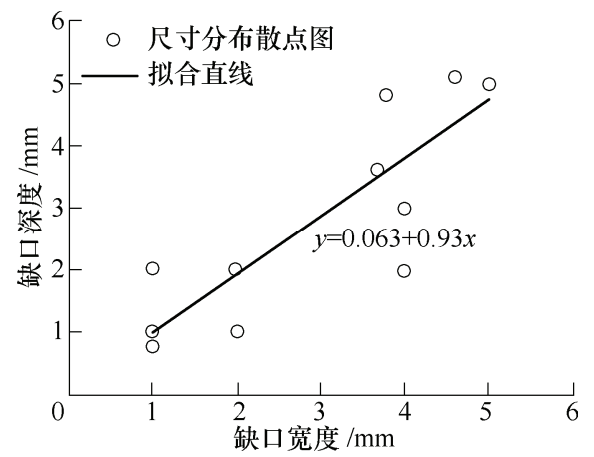

图 16 低压一级叶片缺口损伤宽度和深度散点图

表 9 低压一级叶片缺口宽度与深度聚类分析

\begin{tabular}{cccc}
\hline 类别 & $\begin{array}{c}\text { 缺口宽度 } \\
\text { 平均值 } / \mathrm{mm}\end{array}$ & $\begin{array}{c}\text { 缺口深度 } \\
\text { 平均值 } / \mathrm{mm}\end{array}$ & $\begin{array}{c}\text { 缺口损伤 } \\
\text { 深宽比 }\end{array}$ \\
\hline 1 & 1.40 & 1.36 & 0.97 \\
2 & 4.30 & 4.07 & 0.95 \\
\hline
\end{tabular}

上表可见, 一类缺口的宽度均值为 $1.40 \mathrm{~mm}$, 深度均值为 $1.36 \mathrm{~mm}$, 深宽比为 0.97 ; 另一类缺口 的宽度均值为 $4.30 \mathrm{~mm}$, 深度均值为 4.07 , 深宽比 为 0.95 , 两类缺口的深宽比较为接近。图 16 的拟合 直线为

$$
y=0.063+0.93 x
$$

拟合决定系数 $R^{2}$ 为 0.73 , 拟合程度较好, 直线 斜率和深宽比也较为接近。上述分析表明低压一级 叶片上的缺口损伤宽度与深度尺寸存在较强的线性 正相关，并且缺口损伤宽度越大，深度越深。

\section{5 结论}

通过对四类典型损伤进行分类统计, 并选取了 典型尺寸进行相关性分析, 得到如下结论。

(1) 叶片损伤可以分为缺口、撕裂、凹坑和卷 曲四类，其中缺口损伤所占的比重最大。高、低压 气机间损伤类型差异性不明显。低压压气机叶片损 伤率较高, 并且损伤率随叶片级数的增加呈现下降 趋势; 高压压气机各级叶片中, 损伤类型随级数增 加依次出现缺口、撕裂、凹坑、卷曲等形式, 与叶 片材料以及外来物冲击能量的变化有着密切联系。

(2) 压气机叶片中, 缺口损伤宽度均值大于损 伤深度均值, 且高、低压气机中的缺口损伤尺寸满 足不同的分布形式，压气机级数和损伤尺寸之间并 没有显著的差异性。

(3) 低压压气机叶片上缺口损伤的宽度与深度 相关性不显著, 其缺口按尺寸分布范围可大致分为 两类; 而高压压气机叶片上缺口的宽度与深度存在 显著相关性，其缺口的对数尺寸分布在一定的区间 内; 缺口损伤的位置和缺口宽度没有显著相关性, 而其与缺口深度间有显著相关性, 且缺口距离叶根 越远, 深度尺寸分散程度越大。

(4) 低压压气机第一级叶片大多数缺口损伤位 于距离叶根高度 $80 \%$ 以上区域, 缺口损伤相对位置 与损伤尺寸没有显著的关联, 但缺口损伤宽度和深 度之间具有线性正相关性, 且其散点图存在两个集 中区域。

\section{参 考 文 献}

[1] 关玉㻴, 陈伟, 高德平. 航空发动机叶片外物损伤研究 现状[J]. 航空学报，2007，28(4)：851-857.

GUAN Yupu, CHEN Wei, GAO Deping. Present status of investigation of foreign object damage to blade in aero-engine[J]. Acta Aeronautical et Astronautica Sinica, 2007, 28(4): 851-857.

[2] 胡绪腾. 外物损伤及其对钛合金叶片高循环疲劳强度 的影响[D]. 南京: 南京航空航天大学, 2009 .

HU Xuteng. Foreign object damage and its effect on high cycle fatigue strength of titanium alloy engine blades[D]. Nanjing : Nanjing University of Aeronautics and 
Astronautics, 2009.

[3] 乔文逍, 熊昌炳. 航空发动机外物损伤实验技术研究 [J]. 航空动力学报, 1990, 5(3): 227-228.

QIAO Wenxiao, XIONG Changbing. Experimental technology researches of foreign object damage in aero engine[J]. Journal of Aerospace Power, 1990, 5(3): 227-228.

[4] BRADFORD B. FY01 engine-related mishap summary[J]. Flying Safety, 2002, 58(2): 42-47.

[5] PINKEL B. Factors that affect operational reliability of turbojet engines[J]. Technical Report Archive \& Image Library, 1960, 55(2): 70-71.

[6] 康继东, 陈士煊, 徐志怀, 等. 压气机叶片外物损伤及 其维修性的研究进展 $[\mathrm{J}]$. 燃气涡轮试验与研究, 1998, 11(1): 59-62.

KANG Jidong, CHEN Shixuan, XU Zhihuai, et al. The research progress of foreign object damage and maintainability on compressor blades[J]. Gas Turbine Experiment and Research, 1998， 11(1): 59-62.

[7] 魏雪霞, 李广利. 沙粒对飞机发动机叶片的冲击碰撞 $[\mathrm{J}]$. 北京理工大学学报, 2005, 25(6): 471-474.

WEI Xuexia, LI Guangli. The impact of sand on the blades of airplane engines[J]. Transactions of Beijing Institute of Technology, 2005, 25(6): 471-474.

[8] 葛宁. 发动机叶片抗外物损伤能力评估技术研究[D]. 南京: 南京航空航天大学, 2012.

GE Ning. Research on evaluate technology about resistance to foreign object damage of engine blade[D]. Nanjing: Nanjing University of Aeronautics and Astronautics, 2012.

[9] 林杰威, 张俊红, 张桂昌, 等. 基于连续非线性损伤的 航空发动机叶片疲劳研究[J]. 机械工程学报, 2010, 46(18): $66-70$

LIN Jiewei, ZHANG Junhong, ZHANG Guichang, et al. Study on fatigue damage of aero-engine blade based on non-linear continuum damage model[J]. Journal of Mechanical Engineering, 2010，46(18): 66-70.

[10] 赵振华, 陈伟, 关玉璞, 等. 外物损伤对不锈钢疲劳强 度的影响 [J]. 航空动力学报, 2016, 31(7): 1736-1743. ZHAO Zhenhua, CHEN Wei, GUAN Yupu, et al. Effect of foreign object damage on fatigue strength of stainless steel[J]. Journal of Aerospace Power, 2016, 31(7): 1736-1743.

[11] PETERS J O, RITCHIE R O. Foreign-object damage and high-cycle fatigue of Ti-6Al-4V[J]. Materials Science \& Engineering A, 2013, 319(1): 597-601.

[12] KAUFMAN A, MEYER A J J. Investigation of the effect of impact damage on fatigue strength of jet-engine compressor rotor blades[J]. Technical Report Archive \&
Image Library, 1956, 18(3): 158-162.

[13] THEODORE N. High cycle fatigue: A mechanics of materials perspective[M]. Amsterdam: Elsevier Science\& Technology, 2006.

[14] 潘辉. 叶片外物损伤的特征分析及模拟试验研究 [D]. 南京: 南京航空航天大学, 2012.

PAN Hui. Research on damage characteristics and simulation test of foreign object damage of blade[D]. Nanjing : Nanjing University of Aeronautics and Astronautics, 2012

[15] 马超, 王玉娜, 武耀罡, 等. 航空发动机风扇叶片硬物 冲击损伤特征 [J]. 航空动力学报, 2017，32(5)： 1105-1111.

MA Chao, WANG Yunai, WU Yaogang, et al. Hard object impact damage characteristic of aero-engine fan blade[J] Journal of Aerospace Power, 2017， 32(5): 1105-1111.

[16] 马超, 武耀罡, 师利中, 等. 航空发动机风扇叶片硬物 冲击损伤的统计分析 [J]. 航空维修与工程, 2016(3): 41-42.

MA Chao, WU Yaogang, SHI Lizhong, et al. Hard object impact damage statistical analysis of aero-engine fan blade $[\mathrm{J}]$. Aviation Maintenance and Engineering , 2016(3): 41-42.

[17] 朱红兵, 何丽娟. 在 SPSS10. 0 中进行数据资料正态性 检验的方法 $[\mathrm{J}]$. 首都体育学院学报, 2004, 16(3): 123-125.

ZHU Hongbing, HE Lijuan. Methods of normal test of data in SPSS10.0[J]. Journal of Capital University of Physical Education and Sports, 2004, 16(3): 123-125.

[18] 孙逸敏. 利用 SPSS 软件分析变量间的相关性[J]. 新疆 教育学院学报，2007，23(2): 120-123.

SUN Yimin. Variable correlation analysis based on SPSS software[J]. Journal of Xinjiang Education Institute, 2007, 23(2): 120-123.

[19] 李卫军. K-means 聚类算法的研究综述 [J]. 现代计算 机, 2014(23): 31-32.

LI Weijun. Review of K-mean clustering algorithm[J]. Modern Computer, 2014(23): 31-32.

作者简介: 舒畅, 男, 1995 年出生。主要研究方向为航空发动机结构、 强度与振动。

E-mail: 1575972162@qq.com

程铭, 男, 1982 年出生, 硕士研究生。主要研究方向为航空发动机结构、 强度与振动。

E-mail: 1828433449@qq.com

许显, 男, 1994 年出生, 硕士研究生。主要研究方向为航空发动机结构、 强度与振动。

E-mail:729459449@qq.com

程礼(通信作者), 男, 1963 年出生, 博士, 教授, 博士研究生导师。主 要研究方向为航空发动机故障监控与诊断, 转子动力学。

E-mail: cheng_qiaochu@foxmail.com 